\title{
A checklist of the types of grasses in the Herbarium of the Instituto de Botânica (SP), São Paulo, Brazil
}

\author{
Tatiana Sendulsky ${ }^{1 \dagger}$ and Fábio de Barros ${ }^{1,2}$
}

Received: 02.03.2006; accepted: 23.01.2007

\begin{abstract}
A checklist of the types of grasses in the Herbarium of the Instituto de Botanica (SP), São Paulo, Brazil). A check-list of the types of Poaceae at the herbarium of the Institute of Botany, São Paulo, Brazil (SP) are presented, totalizing 206 specimens and 108 taxa (103 species, two subspecies, and three varieties). Regarding the categories of types, 23 are holotypes, 73 are isotypes, 109 are paratypes, and one is a sintype. Seven type materials pointed in literature as being at the herbarium SP were not found or had an uncertain status.
\end{abstract}

Key words: Gramineae, herbarium, Poaceae, typification.

RESUMO - (Uma listagem dos tipos de gramíneas no Herbário do Instituto de Botânica (SP), São Paulo, Brasil). É apresentada a lista dos materiais tipo de Poaceae depositados no herbário do Instituto de Botânica, São Paulo, Brasil (SP), num total de 206 espécimes, e 108 táxons (103 espécies, duas subspécies e três variedades). Quanto às categorias de tipos, 23 são holótipos, 73 são isótipos, 109 são parátipos e um é um síntipo. Sete materiais-tipo citados na literatura como depositados em SP não foram localizados, ou seu status foi considerado incerto.

Palavras-chave: Gramineae, herbário, Poaceae, tipificação.

\section{Introduction}

A previous list of the types of the Phanerogams of the São Paulo State Herbarium (SP), Instituto de Botânica, São Paulo, Brazil, was published by Mamede (1996), but only eight type collections of Poaceae were mentioned (p. 233). Aditional sources of information about types at the SP herbarium, besides the original descriptions, are the works of Hoehne $(1937,1951)$ where four types of grasses are listed.

During 35 years of intensive research on grasses, the first author found many other type collections that were not referred in the previous types lists. The whole list with 206 type collections is presented here with comments.

\section{Results and Discussion}

The complete list of types of Poaceae housed at the herbarium SP comprises 108 taxa: 103 species, two subspecies and three varieties. Regarding the categories of types, 23 are holotypes, 73 are isotypes, 109 are paratypes, and one is a sintype. Most of the taxa (91) are from Brazil, and only 17 came from other countries. Seven type materials pointed in the literature as being at SP were not found, or their status was regarded as uncertain. The list is presented below.

Types housed at SP:

Altoparadisium chapadense Filg., Davidse, Zuloaga \& Morrone, Ann. Missouri Bot. Gard. 88(2): 363, figs. 1-3. 2001. TYPE: BRAZIL. GoIÁs: Alto Paraíso, Chapada dos Veadeiros, ca. $34 \mathrm{~km} \mathrm{~W}$ of Alto Paraíso, road to Colinas de Goiás, 5-IX-1994, T.S. Filgueiras \& M.L. Fonseca 2987 (holotype IBGE, isotype SP352236).

Filgueiras et al. (2001), referred to the collection locality of the holotype as "ca. $26 \mathrm{~km} \mathrm{~W}$ from Alto Paraíso", but the label of the sheet with the same collector and collection number shows "ca. $34 \mathrm{~km}$ from Alto Paraíso".

Alvimia auriculata Soderstr. \& Londoño, Amer. J. Bot. 75(6): 834, figs. 3-7, 12. 1988. TYPE: BRAZIL. BAHIA: Ilhéus, $16 \mathrm{~km} \mathrm{SW}$ of Olivença, on road to Vila Brasil (ex Ruinha), 21-V-1976, C.E. Calderón et al. 2444 (holotype CEPEC, isotype

1. Instituto de Botânica, Caixa Postal 3005, 01061-970, São Paulo, SP, Brasil

2. Corresponding author: fdebarros@terra.com.br 
SP246680 - 3 sheets). BRAZIL. BAHIA: Ilhéus, $16 \mathrm{~km}$ SW of Olivença, 7-IV-1977, C.E. Calderón 2455 (paratype SP349352).

Alvimia gracilis Soderstr. \& Londoño, Amer. J. Bot. 75(6): 835, figs. 8, 11, 13-16. 1988. TYPES: BRAZIL. BAHIA: Ilhéus, SW of Olivença, on road to Vila Brasil (ex Ruinha), 22-V-1976, C.E. Calderón et al. 2446 (holotype CEPEC, isotype SP337149 - 4 sheets). BRAZIL. BAHIA: Belmonte, $31 \mathrm{~km} \mathrm{SW}$ of Belmonte, 11-IV-1976, C.E. Calderón et al. 2147 (paratype SP349350).

Alvimia lancifolia Soderstr. \& Londoño, Amer. J. Bot. 75(6): 837, figs. 1, 2, 9, 17. 1988. TYPE: BRAZIL. BAHIA: Ilhéus, $16.5 \mathrm{~km} \mathrm{SW}$ of Olivença, Ipiranga Farm, 8-IV-1977, C.E. Calderón 2456 (holotype CEPEC, isotype SP337148 - 3 sheets).

Andropogon brasiliensis A. Zanin \& Longhi-Wagner, Novon 13(3): 368, fig. 1. 2003. TYPES: BRAZIL. Minas Gerais: Bandeira region, $12 \mathrm{~km}$ from Diamantina, S of road Diamantina - Belo Horizonte, 9-13-VII-1977, A.G. Burman 25 (paratype SP199605). BRAZIL. Minas Gerais: Jaboticatubas, km 141 along Lagoa Santa Conceição do Mato Dentro - Diamantina road, 17-IV-1972, A.B. Joly et al. CFSC 1851 (paratype SP183970).

In the original description of the species, Zanin \& Longhi-Wagner (2003) pointed 1851 as the collection number of A.B. Joly in the sheet SP183970, but 1851 is the registration number of the "Coleção Flora da Serra do Cipó" (Collection Flora of the "Cipó" Range), denoted by the abbreviation CFSC, and not the collection number of A.B. Joly himself.

Andropogon monocladus A. Zanin \& LonghiWagner, Novon 13(3): 370, fig. 2. 2003. TYPE: BRAZIL. Distrito Federal: Brasília, Reserva Ecológica do IBGE, 4-VIII-1979, E.P. Heringer et al. 1716 (holotype IBGE, isotype SP219593).

Andropogon reedii Hitchc. \& Ekman ex Hitchc., Misc. Publ. U. S. Dept. Agric. 243: 390. 1936. TYPE: CUBA. CAMAgUeY: Minas, 5-X-1922, E.L. Ekman s.n. (holotype US, isotype SP125044).

Arberella bahiensis Soderstr. \& Zuloaga, Brittonia 37(1): 23, figs. 1-2. 1985. TYPE: BRAZIL. BAHIA: Road Bahia $\mathrm{n}^{\circ}$ 2, $7 \mathrm{~km}$ N of Ibirataia City, Fazenda Santo Antonio, 17-IV-1972, C.E. Calderón \& R.S.
Pinheiro 2224 (holotype CEPEC, isotype SP373370).

Arberella dressleri Soderstr. \& C.E. Calderón, Brittonia 31(4): 433, figs. 1-2. 1979. TYPE: PANAMA. COLÓN: Santa Rita, ca. 25 km before Colón by carretera Transístmica, 9-III-1968, C.E. Calderón 2085 (holotype US, isotype SP224397).

Arthropogon lanceolatus Filg., Bradea 3(36): 307, figs. 3-6. 1982. TYPE: BRAZIL. Minas GeRAIS: Furnas, Ribeirão das Pacas - Cascata, 28-VII-1966, L. Emygdio et al. 2308 (holotype $\mathrm{R}$, isotype SP179350).

Arthropogon rupestris Filg., Nord. J. Bot. 16(1): 69, fig. 1. 1996. TYPE: BRAZIL. GoiÁs: Serra do Caiapó, $17^{\circ} 12^{\prime} \mathrm{S}, 51^{\circ} 47^{\prime} \mathrm{W}$, ca. $30 \mathrm{~km} \mathrm{~S}$ of Caiapônia on roado to Jatai, 26-X-1964, H.S. Irwin \& T.R. Soderstrom 7390 (holotype SP235501).

Arthrostylidium judziewiczii Davidse, Novon 2(2): 81. 1992. TYPE: COSTA RICA. HEREDIA, along River San Rafael, Atlantic slope of Volcán Barva, $10^{\circ} 13^{\prime} \mathrm{N}, 8^{\circ} 05^{\prime} \mathrm{W}, 12-\mathrm{IV}-1986$, M.H. Grayum 7024 (holotype MO, isotype SP332240).

Arundinella montana S.T. Blake, Pap. Dept. Biol. Univ. Queensland 1(18): 16. 1941. TYPE: AUSTRALIA. QUEENSLAND: Moreton District, Mt. Ngungun, 24-II-1940, S.T. Blake 14143 (holotype BRI, isotype SP232473).

Arundoclaytonia dissimilis Davidse \& R.P. Ellis, Ann. Missouri Bot. Gard. 74(3): 479, figs. 1-16. 1987. TYPE: BRAZIL. AMAZONAs, Transamazon Highway, ca. $53 \mathrm{~km} \mathrm{~W}$ of the Aripuanã river, 28-VI-1979, C.E. Calderón et al. 2706 (holotype INPA, isotype SP 373938 - 2 sheets).

Aulonemia chimantaensis Judziewickz \& Davidse, Novon 1(2): 80, fig. 3 G-J. 1991. TYPE: VENEZUELA. Bolívar: Piar, Macizo Del Chimantá, altiplanície en la base meridional de los "farallones" superiores del Apacará-tepui, setor N del macizo, $05^{\circ} 20^{\prime} \mathrm{S}, 06^{\circ} 12^{\prime} \mathrm{W}, 30-\mathrm{I}-1-\mathrm{XII}-1983, \mathrm{~J}$. Steyermark et al. 128369 (holotype MO, isotype SP249398).

Aulonemia fimbriatifolia L.G. Clark, Revista Brasil. Bot. 27(1): 31, fig. 1A-B. 2004. TYPES: BRAZIL. São Paulo: Santo André, Alto da Serra, mata da Estação Biológica, 6-X-1936, F.C. Hoehne \& A. 
Gehrt s.n. (holotype SP36504). BRAZIL. PARANÁ: Quatro Barras, Serra da Graciosa, along trail to Morro Mãe Catira, above a creek flowing into the Corvo river, $25^{\circ} 15^{\prime} \mathrm{S}, 48^{\circ} 50^{\prime} \mathrm{W}, 18-\mathrm{III}-1991$, L.G. Clark et al. 936 (paratype SP249443). BRAZIL. PARANá: Quatro Barras, morro Sete, Serra do Mar, 2520'S, 4852'W, 9-III-1994, L.G. Clark et al. 1195 (paratype SP367177). BRAZIL. SANTA Catarina: Campo Alegre, along the Negro river, Serra do Iquererim, about 19,5 km from Postema, 2603'S, 4903'W, 16-III-1991, L.G. Clark \& W. Oliveria 926 (paratype SP249432). BRAZIL. SÃo Paulo: Biritiba Mirim, Estação Biológica de Boracéia, along Claro river, Pouso Verde, 24-II-1991, 23³6'S, 4558'W, L.G. Clark \& M. Morel 824 (paratype SP252412). BRAZIL. SÃo Paulo: Santo André, Paranapiacaba, Estação Biológica, 29-VI-1948, M. Kuhlmann 3137 (paratype SP153821). BRAZIL. SÃo PAULO: Santo André, Paranapiacaba, Estação Biológica, 18-III-1948, M. Kuhlmann 3138 (paratype SP153822). BRAZIL. São Paulo: Santo André, São Paulo - Santos railway, Estação Biológica, 29V-1969, J. Mattos 15444 (paratype SP111147). BRAZIL. São Paulo: São José do Barreiro, Serra da Bocaina, Campos da Bocaina, Rio das Pedras, Cachoeira das Meninas, $22^{\circ} 45^{\prime} \mathrm{S}, 4^{\circ} 47^{\prime} \mathrm{W}$, 4-III-1992, L.G. Clark \& P.G. Windisch 1060 (paratype SP358570).

Axonopus grandifolius Renvoize, Kew Bull. 39(1): 183. 1984. TYPE: BRAZIL. BAHIA: Rio Cumbuca, about $3 \mathrm{~km} \mathrm{~N}$ of Mucuge on the Andaraí road, ca. $13^{\circ} 00^{\prime}$ S, 41 ${ }^{\circ} 23^{\prime}$ W, 5-II-1974, R.M. Harley 16012 (holotype CEPEC, isotype SP224695).

Bulbulus nervatus Swallen, Phytologia 11(3): 154. 1964 (= Rehia nervata (Swallen) Fijten, Blumea 22(3): 416. 1975). TYPE: BRAZIL. MARANHÃO: from Caxias to Barra do Corda, 18-26-II-1934, J.R. Swallen 3552 (holotype US, isotype SP337147).

Catabrosa burkartii Hitchc., J. Wash. Acad. Sci. 24: 481. 1934. (= Poa perligulata Pilg.). TYPE: ARGENTINA. Tucumán: Tafi, summit of Calchaquíes, 30-I-1933, A. Burkart 5348 (holotype US, isotype SP30545).

Centrochloa singularis Swallen, J. Wash. Acad. Sci. 25(4): 192. 1935. TYPES: BRAZIL. MARANHÃo: between Barra do Corda and Grajaú, 1-5-III-1934, J.R. Swallen 3703 (holotype US, isotype
SP35891). BRAZIL. Goiás: Philadelphia, 19-III-1934, J.R. Swallen 3921 (paratype SP35890). BRAZIL. MARANHÃO: between Carolina and Riachão, 20-25-III-1934, J.R. Swallen 4021 (paratype SP35889).

Chusquea caparaoensis L.G. Clark, Brittonia 44(4): 408, fig. 9H, I. 1992. TYPE: BRAZIL. Minas GERAIS: Caparaó, Parque Nacional do Caparaó, trail to Pico da Bandeira, just above turnoff to Vale

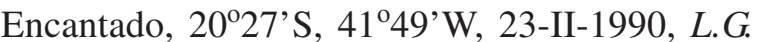
Clark \& M. Morel 701 (holotype SP238298 - 2 sheets).

Chusquea erecta L.G. Clark, Brittonia 44(4): 397, fig. 1. 1992. TYPE: BRAZIL. São PAULo: Salesópolis, Reserva Hidrológica do Rio Claro-SABESP/ Estação Biológica Boracéia, Campos da Boracéia,

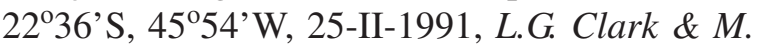
Morel 826 (holotype SP252414 - 2 sheets).

Chusquea longispiculata L.G. Clark, Revista Brasil. Bot 27(1): 34, fig. 1 C-F. 2004. TYPE: BRAZIL. São Paulo: Sete Barras, XI-1977, D.M. Vital s.n. (holotype SP217610 - 4 sheets). BRAZIL. Rio DE JANEIRO: Rio de Janeiro, Estrada Dona Castourina, caminho para Vista Chinesa, Parque Nacional da

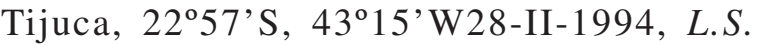
Sarahyba et al. 1064 (paratype SP373514). BRAZIL. São Paulo: Parque Estadual Carlos Botelho, SP 139, $65 \mathrm{~km}$ from BR 116 on road from Sete Barras to S. Miguel Arcanjo, $24^{\circ} 05^{\prime}$ S, 4759'W16-II-1992, L.G. Clark \& W Oliveira 1026 (paratype SP373316). BRAZIL. São PAULO: Santo André, Paranapiacaba, 29-IV-1948, M. Kuhlmann 3140 (paratype SP153828). BRAZIL. São Paulo: Santo André, Paranapiacaba, Estação Biológica, Barroca das Onças e picada Washington Luiz, 10-VI-1948, M. Kuhlmann 3141 (paratype SP153843). BRAZIL. SÃo PAULO: SP 139, km 78-79, Serra de Paranapiacaba, Reserva Florestal Carlos Botelho, just before river Taquaral, $24^{\circ} 01$ 02'S, 4801-02'W, 28-I-1990, L.G. Clark \& P.G. Windisch 645 (paratype SP238238).

Chusquea mimosa var. australis L.G. Clark, Brittonia 44(4): 414, fig. 14 G-I. 1992. TYPE: BRAZIL. Rio Grande do Sul: BR 116, between Caxias do Sul and Vacaria, about $20.5 \mathrm{~km} \mathrm{~S}$ of San Marcos, 2907'S, 51 08' W, 15-III-1991, L.G. Clark \& W. Oliveira 919 (paratype SP249424). BRAZIL. SAnta Catarina: along road to Morro da Igreja, 
$8.9 \mathrm{~km}$ from the main road between Urubici and Tubarão, Serra Geral, $28^{\circ} 06^{\prime} \mathrm{S}, 49^{\circ} 28^{\prime} \mathrm{W}$, 5-III-1991, L.G. Clark \& P.G. Windisch 868 (paratype SP249491). BRAZIL. SANTA CatARINA: Bom Jardim, Morro do Trombudo, 12-XII-1958, R. Reitz \& R.M. Klein 7824 (paratype SP113031). BRAZIL. Santa Catarina: Bom Retiro, Campo dos Padres Farm, Campo dos Padres, 17-19-XI-1956, L.B. Smith et al. 7773 (paratype SP235773). BRAZIL. SANTA CATARINA: road from Orleans to Bom Jardim da Serra, 28 $22^{\prime} \mathrm{S}, 4^{\circ} 33^{\prime} \mathrm{W}$, 24-II-1992, L.G. Clark et al. 1044 (paratype SP373720). BRAZIL. Santa Catarina: Serra Geral, road from Timbé do Sul to S. José dos Ausentes, about $15.2 \mathrm{~km}$ from Timbé do Sul, Serra da Rocinha (Serra do Pinheiro), 2849'S, 4956'W, 6-III-1991, L.G. Clark \& P.G. Windisch 875 (paratype SP249516).

Chusquea nudiramea L.G. Clark, Brittonia 44(4): 415, figs. 15. 1992. TYPE: BRAZIL. SANTA CATARINA: Santo Amaro da Imperatriz, Rio Cobrinha de Ouro, about 50-75 $\mathrm{m}$ up the river from the rapids,

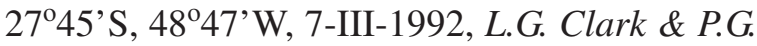
Windisch 1068 (paratype SP372299).

Chusquea nutans L.G. Clark, Brittonia 44(4): 398, figs. 3A-F, 4, 5. 1992. TYPE: BRAZIL. MinAS GERAIS: Brumadinho, ca. $16 \mathrm{~km}$ from Belo Horizonte along BR 040, Condomínio Retiro das Pedras, below rua Quaresmeira Roxa, drainage of rio Mutuca, periphery of Serra da Moeda, $20^{\circ} 07^{\prime} \mathrm{S}, 43^{\circ} 58^{\prime} \mathrm{W}$, 29-I-1991, L.G. Clark \& et al. 768 (paratype SP252357). BRAZIL. MINAS GERAIS: Brumadinho, ca. $16 \mathrm{~km}$ from Belo Horizonte along BR 040, Condomínio Retiro das Pedras, below rua Quaresmeira Roxa, drainage of rio Mutuca, periphery of Serra da Moeda, $20^{\circ} 07^{\prime} \mathrm{S}, 43^{\circ} 58^{\prime} \mathrm{W}$, 29-I-1991, L.G. Clark et al. 769 (paratype SP252358). BRAZIL. Minas Gerais: Caeté, Serra da Piedade, 1949'S, 4340'W, 1-III-1990, L.G. Clark \& M. Morel 719 (paratype SP238280). BRAZIL. Minas Gerais: Caeté, Serra da Piedade, 1949'S, 4340'W, 1-III-1990, L.G. Clark \& M. Morel 764 (paratype SP252353). BRAZIL. MINAS Gerais: Parque Natural e Santuário do Caraça, along the creek that flows into rio Caraça, near the Piscina, $1^{\circ} 58^{\prime}$ 'S, $43^{\circ} 22^{\prime} \mathrm{W}, 28-\mathrm{II}-1990$, L.G. Clark \& M. Morel 715 (paratype SP238296). BRAZIL. Minas Gerais: Serra do Garimpo, 13-I-1921, F.C. Hoehne s.n. (paratype SP4954).
Chusquea pulchella L.G. Clark, Novon 3(3): 236, fig. 3 F-J. 1993. TYPE: BRAZIL. São PAulo: BR-116, ca. km 517, heading N to São Paulo, Rio Braço Feio, ca. $60 \mathrm{~km}$ before the spring, $48^{\circ} 19^{\prime} \mathrm{W}$, 245'S, 19-III-1991, L.G. Clark \& W. Oliveira 939 (holotype SP249398). BRAZIL. São PaUlo: BR-116, ca. km 517, heading N to São Paulo, Rio Braço Feio, ca. $60 \mathrm{~km}$ before spring, $48^{\circ} 15^{\prime} \mathrm{W}$, 245' S, 4-III-1990, L.G. Clark \& P.G. Windisch 726 (paratype SP238267). BRAZIL. São PAULO: ca. $38 \mathrm{~km} \mathrm{SW}$ of Jacupiranga, along highway 116 to Curitiba, 8-III-1976, G. Davidse \& W.G. D'Arcy 10950 (paratype SP160200).

Chusquea riosaltensis L.G. Clark, Brittonia 44(4): 403, fig. 6. 1992. TYPE: BRAZIL. Minas Gerais: Lima Duarte, Serra do Ibitipoca, Parque Estadual (Florestal) do Ibitipoca, Paredão do Rio Salto, fork from trail to Ponte da Pedra, 2-II-1991, L.G. Clark \& M. Morel 775 (holotype SP252364).

Chusquea windischii L.G. Clark, Brittonia 44(4): 405, fig. 9A-G. 1992. TYPE: BRAZIL. SANTA CATARINA: Urubici, Morro da Igreja, Serra Geral, 28 $07^{\prime}$ 'S, 49²9'W, 25-II-1992, L.G. Clark et al. 1046 (holotype SP373310). BRAZIL. SANTA Catarina: along road to Morro da Igreja, about $16.7 \mathrm{~km}$ from the main road between Urubibi and Tubarão, above Caiambora Farm, Serra Geral, $28^{\circ} 07^{\prime}$ S, 49 $29^{\prime} \mathrm{W}$, 5-III-1991, L.G. Clark \& P.G. Windisch 869A (paratype SP249493). BRAZIL. SANTA Catarina: Urubici, Morro da Igreja, Serra Geral, 28 $07^{\circ} \mathrm{S}$, 49²9'W, 25-II-1992, L.G. Clark et al. 1047 (paratype SP373309).

Coix gasteenii B.K. Simon, Austrobaileya 3(1): 1, figs. 1-3. 1989. TYPE: AUSTRALIA. Queensland: Cook, 15¹8'S, 144³6'E, Cabbage Tree creek, 19 $\mathrm{km}$ E of Old Laura on road to Cooktown, 13-V-1987, J.R. Clarkson \& B.K. Simon 7215 (holotype BRI, isotype SP246848).

Criciuma asymmetrica Soderstr. \& Londoño, Amer. J. Bot. 74(1): 35, figs. 1, 2, 5a, b. 1987. TYPE: BRAZIL. BAHIA: Una, Road Una-Olivença, ca. 12 $\mathrm{km} \mathrm{N}$ of Una city, Farm of Clovis de Matos Pires, 26-II-1979, C.E. Calderón \& T.S. Santos 2486 (holotype CEPEC, isotype SP337150).

Diandrolyra tatianae Soderstr. \& Zuloaga, Brittonia 37(1): 2, fig. 1. 1985. TYPE: BRAZIL. São PAULO: Ubatuba, $15 \mathrm{~km}$ NE of Ubatuba, near Lázaro 
beach, 23⒉ ${ }^{\circ} \mathrm{S}, 4^{\circ} 55^{\prime} \mathrm{W}, 16-\mathrm{V}-1972$, T.R. Soderstrom \& T. Sendulsky 1993 (holotype SP373369).

Eriochrysis glabrifolia Swallen, Phytologia 14(2): 90. 1966 (= Eriochrysis cayanensis Beauv.). TYPE: BRAZIL. Paraná: Ponta Grossa, Estação Experimental, 28-I-1946, J.R. Swallen 8296 (holotype US, isotype SP337153).

Festuca talamancensis Davidse, Novon 2(4): 324, fig. 1E-F. 1992. TYPE: COSTA RICA. SAN JosE: Cartago, Cordillera de Talamanca, cerros Cuerici, Parque Nacional Chirripó, $09^{\circ} 35^{\prime} \mathrm{N}, 83^{\circ} 38^{\prime} \mathrm{W}$, 17-IX-1983, G. Davidse 24776 (holotype MO, isotype SP358456).

Ichnanthus ramosissimus K.E. Rogers, Phytologia, 24(5): 416. 1972. (= Ichnanthus ruprechtii Doell). TYPE: BRAZIL. Santa Catarina: Rio Caçador, 22-I-1946, J.R. Swallen 8293 (holotype US, isotype SP337142).

Lithachne horizontalis Chase, J. Wash. Acad. Sci. 25(4): 189, fig. 1. 1935. TYPE: BRAZIL. MINAS GERAIS: near Belo Horizonte, 25-III-1925, A. Chase 9057 (holotype US, isotype SP35991).

Luziola fragilis Swallen, Ann. Missouri Bot. Gard. 52(3): 474. 1965. TYPE: BRAZIL. MAto Grosso: Aquidauana, 24-VI-1946, J.R. Swallen 9538 (holotype US, isotype SP337151).

Maclurolyra tecta Calderón \& Soderstr., Smithsonian Contr. Bot. 11: 6, figs. 4-7. 1973. TYPE: PANAMA. Colón: Santa Rita, ca. 25 km before Colón by the carretera Transistmica, 5-III-1968, C.E. Calderón 2080 (paratype SP224383).

Merostachys abadiana Send., Novon 5(1): 77, fig. 1. 1995. TYPE: BRAZIL. SÃo PAULO: Itatinga, Abadia, 26-IX-1978, O. Campos Neto s.n. (holotype SP154498).

Merostachys annulifera Send., Novon 7(3): 286, fig. 1. 1997. TYPES: BRAZIL. BAHIA: Uruçuca, road Serra Grande-Ilhéus, km 2, 20-X-1983, T.S. Santos et al. 3906 (holotype SP236268 - 2 sheets). BRAZIL. BAHIA: Ibirataia, $23 \mathrm{~km} \mathrm{~N}$ of Ibirataia, on side road connecting with BR 101, Macedonia Farm, 6-IV-1976, C.E. Calderón et al. 2364 (paratype SP367112 - 2 sheets). BRAZIL. BAHIA: Ituberá, ca. $2 \mathrm{~km}$ from Ituberá on road to Gandú,
Inferno Verde Farm, 23-IV-1976, T.R. Soderstrom et al. 2158 (paratype SP235902 - 2 sheets). BRAZIL. BAHIA: Una, $19 \mathrm{~km}$ from Uma on road to Santa Luzia do Salobro, 27-V-1976, T.R. Soderstrom et al. 2232 (paratype SP235909). BRAZIL. BAHIA: Una, road Una - São José do Macuco, 18 km NW of Una, 29-IV-1976, C.E. Calderón et al. 2405 (paratype SP367113 - 2 sheets). BRAZIL. BAHIA: Una, km 16,8 on the old road Una - Olivença, 18-III-1992, X. Londoño et al. 730 (paratype SP373513 - 2 sheets).

Merostachys argentea Send., Novon 7(3): 287, fig. 2. 1997. TYPE: BRAZIL. BAHIA: Porto Seguro, forested slope of hill on Carvalho Farm, ca. $26 \mathrm{~km}$ W of town of Monte Pascoal, 15-V-1976, T.R. Soderstrom et al. 2213 (holotype CEPEC, isotype SP235912 - 2 sheets).

Merostachys bifurcata Send., Novon 7(3): 290, fig. 3. 1997. TYPES: BRAZIL. BAHIA: Porto Seguro, Monte Pascoal National Park, 14 km E of BR 101 at a point $13 \mathrm{~km} \mathrm{~N}$ of Itamarajú, 12-V-1976, T.R. Soderstrom et al. 2202 (holotype US, isotype SP235915 - 2 sheets). BRAZIL. BAHIA: Belmonte, Reserva Gregório Bondar of CEPLAC's Experimental Station, 10-IV-1976, T.R. Soderstrom et al. 2138 (paratype SP235913).

Merostachys brevigluma Send., Kew Bull. 56(3): 629, fig. 2. 2001. TYPES: BRAZIL. MinAS GERAIS: São Francisco dos Campos, Delfim Moreira, São Francisco Farm, 8-VI-1950, M. Kuhlmann 2453 (holotype SP153837). BRAZIL. São PAULO: Campos de Jordão, 4-X-1948, M. Kuhlmann s.n. (paratype SP79758). BRAZIL. São PaUlo: Campos de Jordão, Alto da Ferradura, 4-I-1981, W. Bockmann s.n. (paratype SP169274 - 2 sheets). BRAZIL. São PAULO: Campos de Jordão, Umuarama, 22-XI-1949, M. Kuhlmann 2084 (paratype SP153838). BRAZIL. São PAULO: São José dos Campos, Santa Cruz Farm, 14-IV-1981, M.T.P. Azevedo s.n. (paratype SP169276).

Merostachys burmanii Send., Novon 2(2): 111, fig. 1. 1992. TYPES: BRAZIL. SÃo PAULO: São Paulo, Jardim Botânico, 25-III-1944, M. Kuhlmann s.n. (holotype SP79757). BRAZIL. Rio DE JANEIRO: Rio de Janeiro, Campo Grande, Serra do Mendanha, 4-IV-1978, G. Martinelli et al. 4129 (paratype SP236259). BRAZIL. Rio DE JANEIRO: Serra dos Órgãos, Fazenda Dr. Portugal, 27-XI-1948, A.C. 
Brade 19457 (paratype SP236258). BRAZIL. São PAulo: São Paulo, Jardim Botânico, 19-I-1944, J.F. Toledo s.n. (paratype SP50362). BRAZIL. São PAulo: São Paulo, Jardim Botânico, 23-III-1944, A. Gehrt \& M. Kuhlmann s.n. (paratype SP50462). BRAZIL. São PAULO: São Paulo, Jardim Botânico, picada Porsh, lago das Guaricangas, 19-V-1959, J.F. Toledo s.n. (paratype SP23849 - 3 sheets).

Merostachys calderoniana Send., Novon 7(3): 290, fig. 4. 1997. TYPES: BRAZIL. BAHIA: border of Municipalities of Camacã and Mascote, $24 \mathrm{~km} \mathrm{~S}$ of Camacã on BR 101, 8-IV-1976, T.R. Sodestrom et al. 2130 (paratype SP235906). BRAZIL. BAHIA: Jaguaquara, ca. $9.5 \mathrm{~km}$ SE of Jaguaquara City, Mundo Novo Farm, 13-IV-1976, C.E. Calderón et al. 2380 (paratype SP333944). BRAZIL. BAHIA: Una, km 35 road Olivença - Una, $1.5 \mathrm{~km}$ by a left branch, 17-III-1992, X. Londoño et al. 724 (paratype SP258123). BRAZIL. BAhIA: Una, "Reserva Biológica do Mico-Leão", entrance at $\mathrm{km} 46$ of BA 001 Ilhéus to Una, about $0.6 \mathrm{~km}$ from the main gate, $15^{\circ} 09^{\prime} \mathrm{S}, 39^{\circ} 05^{\prime} \mathrm{W}, 6-\mathrm{II}-1994$, A.M. Carvalho et al. 4370 (paratype SP373943).

Merostachys caucaiana Send., Novon 5(1): 80, fig. 2. 1995. TYPE: BRAZIL. São Paulo: Cotia, Caucaia, 1978, N. Menezes \& O. Handro s.n. (holotype SP262720 - 3 sheets).

Sendulsky (1995) pointed the herbarium sheet $O$. Handro \& N. Menezes s.n. (SP262720) as holotype, but in the label of this particular sheet it is written $N$. Menezes \& O. Handro s.n.

Merostachys filgueirasii Send., Novon 5(1): 80, fig. 3. 1995. TYPES: BRAZIL. Distrito Federal: Catetinho, 7-I-1982, T.S. Filgueiras \& B.A.S. Pereira 953 (holotype IBGE, isotype SP212577). BRAZIL. Distrito Federal: Reserva Ecológica do IBGE, mata do Ribeirão Taquara, 20-XI-1990, T.S. Filgueiras \& A.L. Brochado 2009 (paratype SP246657 - 8 sheets).

Merostachys lanata Send., Novon 7(3): 292, fig. 5. 1997. TYPES: BRAZIL. BAHIA: Porto Seguro, $7 \mathrm{~km} \mathrm{~W}$ of Porto Seguro on road to Eunápolis, 9-V-1976, T.R. Soderstrom et al. 2191 (holotype US, isotype SP235911 - 3 sheets). BRAZIL. BAHIA: Belmonte, 27 km SW of Belmonte, 11-IV-1976, T.R. Sodestrom et al. 2146 (paratype SP235895).
BRAZIL. BAHIA: Porto Seguro, Parque Nacional Monte Pascoal, $14 \mathrm{~km}$ E of BR 101 at a point 13 $\mathrm{km} \mathrm{N}$ of Itamaraju, 13-V-1976, T.R. Sodestrom et al. 2207 (paratype SP235905 - 2 sheets). BRAZIL. BAHIA: Santa Cruz de Cabrália, 9 km SW of Santa Cruz de Cabrália on road to Porto Seguro, 9-V-1976, T.R. Sodestrom et al. 2192 (paratype SP235904 - 2 sheets).

Merostachys leptophylla Send., Novon 7(3): 295, fig. 6. 1997. TYPES: BRAZIL. BAHIA: Ibicaraí, between Ibicaraí and Floresta Azul, at $7 \mathrm{~km}$ of Ibicaraí, 3-IV-1976, T.R. Soderstrom et al. 2106 (holotype US, isotype SP235893). BRAZIL. BAHIA: Porto Seguro, Parque Nacional de Monte Pascoal, Monte Pascoal, ca. 165'' S, 39²4'W, 6-IV-1972, C.E. Calderón \& R.S. Pinheiro 2202 (paratype SP224363). BRAZIL. BAHIA: Porto Seguro, Parque Nacional Monte Pascoal, $14 \mathrm{~km}$ E of BR 101 at a point $13 \mathrm{~km} \mathrm{~N}$ of Itamaraju, 12-V-1976, T.R. Sodestrom et al. 2204 (paratype SP235914 - 2 sheets).

Merostachys magellanica Send., Novon 5(1): 86, fig. 6. 1995. TYPES: BRAZIL. São Paulo: Anhembi, Barreiro Rico Farm, 2040'S, 4809'W, 11-I-1988, J.C. Reis-de-Magalhães s.n. (holotype SP248339). BRAZIL. São Paulo: Anhembi, Barreiro Rico Farm, 21-II-1956, J.C. Reis-deMagalhães s.n. (paratype SP248383). BRAZIL. São Paulo: Cananéia, X-1956, C. Pereira s.n. (paratype SP169270). BRAZIL. SÃo PaUlo: Cananéia, Parque Estadual da Ilha do Cardoso, 8-XII-1987, M. Kirizawa 1984 (paratype SP220844 - 4 sheets). BRAZIL. São PAulo: Cananéia, Ilha do Cardoso, Cambriu, 23-VIII-1988, L. Rossi et al. 486 (paratype SP238022). BRAZIL. São Paulo: Cananéia, Parque Estadual da Ilha do Cardoso, 3-XII-1990, F. Barros 2024 (paratype SP246679 - 4 sheets). BRAZIL. São Paulo: Iguape, 19-XI-1987, E.L.M. Catharino 1334 (paratype SP237817).

Merostachys medullosa Send., Novon 7(3): 298, fig. 8. 1997. TYPES: BRAZIL. BAHIA: Porto Seguro, Carvalho Farm, ca. $26 \mathrm{~km} \mathrm{~W}$ of town of Monte Pascoal, at end of road that intersects with BR 101 in Monte Pascoal, 15-V-1976, T.R. Soderstrom et al. 2212 (holotype US, isotype SP235900 - 2 sheets). BRAZIL. BAHIA: Ubaíra, 2 km E of Ubaira on road to Mutuipe, Pindaba de Dentro Farm, 
27-IV-1976, T.R. Sodestrom et al. 2166 (paratype SP235916 - 2 sheets).

Merostachys procerrima Send., Novon 7(3): 300, fig. 9. 1997. TYPES: BRAZIL. BAHIA: Porto Seguro, Reserva Biológica do Pau Brasil (CEPLAC), 7-V-1976, T.R. Sodestrom et al. 2187 (holotype US, isotype SP235919 - 2 sheets). BRAZIL. BAHIA: Camamu, $42 \mathrm{~km}$ of Camamu on road to Ubaitaba, 22-IV-1976, T.R. Sodestrom et al. 2157 (paratype SP235903). BRAZIL. BAHIA: Porto Seguro, Parque Nacional Monte Pascoal, $14 \mathrm{~km}$ E of BR 101 at a point $13 \mathrm{~km} \mathrm{~N}$ of Itamaraju, 11-V-1976, T.R. Sodestrom et al. 2198 (paratype SP235896).

Merostachys ramosissima Send., Novon 7(3): 300, fig. 10. 1997. TYPE: BRAZIL. BAHIA: Ubaira, $3 \mathrm{~km}$ E of Ubaira and $11 \mathrm{~km} \mathrm{~W}$ of Mutuipe, 26-IV-1976, T.R. Soderstrom et al. 2164 (holotype US, isotype SP235920 - 2 sheets).

Merostachys scandens Send., Novon 5(1): 92, fig. 9. 1995. TYPES: BRAZIL. São PAULO: São Paulo, $10 \mathrm{~km} \mathrm{~S}$ of center of city of São Paulo, in grounds of the Instituto de Botânica, Parque do Estado, 6-XII-1973, T. Sendulsky 1319 (holotype SP166800). BRAZIL. São Paulo: São Paulo, Jardim Botânico, 1-XII-1941, O. Handro s.n. (paratype SP79756). BRAZIL. São PaUlo: São Paulo, $10 \mathrm{~km}$ S of center of city of São Paulo, in grounds of the Instituto de Botânica, Parque do Estado, 6-XII-1973, T. Sendulsky 1317 (paratype SP166797). BRAZIL. São PaUlo: São Paulo, 10 km $S$ of center of city of São Paulo, in grounds of the Instituto de Botânica, Parque do Estado, 6-XII-1973, T. Sendulsky 1320 (paratype SP166799 - 3 sheets). BRAZIL. SÃo PAULO: São Paulo, $10 \mathrm{~km} \mathrm{~S}$ of center of city of São Paulo, in grounds of the Instituto de Botânica, Parque do Estado, 17-XII-1973, T. Sendulsky 1323 (paratype SP166803 - 2 sheets). BRAZIL. São PAUlo: São Paulo, Reserva Biológica do Parque Estadual das Fontes do Ipiranga, 8-III-1974, T. Sendulsky 1365 (paratype SP174491).

Merostachys skvortzovii Send., Novon 5(1): 94, fig. 10. 1995. TYPES: BRAZIL. São PAulo: São Paulo, Parque do Estado and Jardim Botânico, 20-X-1973, B. Skvortzov s.n. (holotype SP120954). BRAZIL. SÃo PaUlo: São Paulo, $10 \mathrm{~km} \mathrm{~S}$ of center of city of São Paulo, in grounds of the Instituto de Botânica, Parque do Estado, 10-X-1973, T. Sendulsky 1314 (paratype SP166801 - 2 sheets). BRAZIL. São Paulo: São Paulo, $10 \mathrm{~km}$ S of center of city of São Paulo, in grounds of the Instituto de Botânica, Parque do Estado, 20-X-1973, T. Sendulsky 1315 (paratype SP166798). BRAZIL. São PAULO: São Paulo, $10 \mathrm{~km} \mathrm{~S}$ of center of city of São Paulo, in grounds of the Instituto de Botânica, Parque do Estado, 6-XII-1973, T. Sendulsky 1318 (paratype SP166796). BRAZIL. São Paulo: São Paulo, $10 \mathrm{~km} \mathrm{~S}$ of center of city of São Paulo, in grounds of the Instituto de Botânica, Parque do Estado, 17-XII-1973, T. Sendulsky 1321 (paratype SP169753). BRAZIL. São Paulo: São Paulo, 10 km $S$ of center of city of São Paulo, in grounds of the Instituto de Botânica, Parque do Estado, 17-XII-1973, T. Sendulsky 1322 (paratype SP166795). BRAZIL. São Paulo: São Paulo, 10 $\mathrm{km} \mathrm{S}$ of center of city of São Paulo, in grounds of the Instituto de Botânica, Parque do Estado, 23-XII-1973, T. Sendulsky 1324 (paratype SP166802 - 3 sheets). BRAZIL. São PAULO: São Paulo, Jardim Botânico, 21-X-1941, A. Gehrt s.n. (paratype SP46009). BRAZIL. São PaUlo: São Paulo, Reserva Biológica do Parque Estadual das Fontes do Ipiranga, 7-II-1974, T. Sendulsky 1341 (paratype SP174478 - 2 sheets).

Mesosetum alatum Filg., Brittonia 39(2): 306, fig. 1 A-B. 1987. TYPE: BRAZIL. Mato Grosso: Xavantina - Cachimbo road, $65 \mathrm{~km}$ from Xavantina, 24-V-1966, D.R. Hunt \& J. Ferreira-Ramos 5552 (holotype UB, isotype SP114394).

Mesosetum pittieri Hitchc., Proc. Biol. Soc. Wash. 40: 85. 1927. TYPE: PANAMA. PANAMA: Sabana de Juan Corso, near Chepó, 11-X-1911, H. Pittier 4516 (paratype SP223601).

Ophiochloa hydrolithica Filg., Davidse \& Zuloaga, Novon 3(4): 360, fig. 1-3. 1993. TYPE: BRAZIL. GoIÁs: Niquelândia, Macedo, ca. $20 \mathrm{~km} \mathrm{~N}$ of Niquelândia, 4-VIII-1992, T.S. Filgueiras \& R.D. Lopes 2438 (holotype SP358455). BRAZIL. GoIÁs: Niquelândia, Macedo, ca. $20 \mathrm{~km} \mathrm{~N}$ of Niquelândia, 14¹8'S, 48²3'W, 19-V-1993, T.S. Filgueiras 2466 (paratype SP373937).

In the original description of Filgueiras et al. (1993a) the herbarium material indicated as paratype is T.S. Filgueiras \& F.C.A. Oliveira 2466 instead of T.S. Filgueiras 2466, but it is quite sure that the sheet SP373937 is a paratype as written in its label. 
Otachyrium grandiflorum Send. \& Soderstr., Smithsonian Contrib. Bot. 57: 7, fig. 2. 1984. TYPE: BRAZIL. GoIÁs: Alto Paraiso, Chapada dos Veadeiros, 9-X-1979, E.P. Heringer et al. 2381 (paratype SP373368).

Otachyrium piligerum Send. \& Soderstr., Smithsonian Contr. Bot. 57: 9, fig. 3. 1984. TYPE: BRAZIL. GoIÁs: Formosa, $24 \mathrm{~km}$ N of Vila JK, $\mathrm{km} 147$ of road BR $020,15^{\circ} 02^{\prime} \mathrm{S}, 47^{\circ} 04^{\prime} \mathrm{W}$, 10-VI-1981, J.F.M. Valls et al. 6009 (holotype CEN, isotype SP358459).

Otatea aztecorum (McClure \& E.W. Sm.) Calderón \& Soderstr., Smithsonian Contr. Bot. 44: 21. 1980 $\equiv$ Yushania aztecorum McClure \& E.W. Sm., Smithsonian Contr. Bot. 9: 116, figs. 46-47. 1973. 三 Otatea acuminata (Munro) C.E. Calderón \& Soderstr. subsp. aztectorum (McClure \& E.W. Sm.) R. Guzman, Anaya \& Santana-Mich., Bol. Inst. Bot. Univ. Guadalajara 5(10): 4. 1984. TYPE: MÉXICO. Sinaloa: near Rosario, la Dispensa, 5-VIII-1943, F.A. McClure 21204 (holotype US, isotype SP224367 - 2 sheets).

Panicum albospiculatum Swallen, Sellowia 18: 110. 1966. (= Panicum rude Nees). TYPE: BRAZIL. Santa Catarina: Rio Caçador, 22-I-1946, J.R. Swallen 8291 (holotype US, isotypes SP337143, SP373934). BRAZIL. Santa Catarina: Rio do Sul, Alto Matador, 16-X-1958, R. Reitz \& R.M. Klein 7269 (paratype SP373933).

Panicum blepharophorum Mez, Bot. Jahrb. 56(125): 4. 1921. (= Panicum piauiense Swallen). TYPE: BRAZIL. PIAUI: G. Gardner 2016 (holotype B, isotype SP373935 - fragment).

The material housed at SP is a fragment of the isotype from $\mathrm{W}$.

Panicum carautae Renvoize, Kew Bull. 32(2): 422. 1978. TYPE: BRAZIL. Rio DE JANeIRo: Parque Nacional da Tijuca, Pedra da Gávea, 3-VIII-1975, J.P.P. Carauta 1713 (holotype $\mathrm{K}$, isotype SP131231).

Panicum cervicatum Chase ex Luces, J. Wash. Acad. Sci. 32(6): 164, fig. 10. 1942. TYPE: BRAZIL. Mato Grosso do Sul (Mato Grosso): Três Lagoas, 4-5-II-1930, A. Chase 10737 (holotype US, isotype SP52979).
Panicum cipoense Renvoize \& Send., Kew Bull. 35(1): 229, fig. 1. 1980. TYPES: BRAZIL. MinAs GERAIS: Jaboticatubas, along road Lagoa SantaConceição de Mato Dentro-Diamantina, 10-XII-1971, T. Sendulsky et al. 419 (holotype SP165678). BRAZIL. Minas Gerais: Jaboticatubas, Serra do Cipó, 9-XII-1971, T. Sendulsky et al. 389 (paratype SP165679). BRAZIL. Minas Gerais: Jaboticatubas, Serra do Cipó, km 128 along road Lagoa Santa - Conceição do Mato Dentro - Diamantina, 5-III-1972, A.B. Joly et al. CFSC 1112 (paratype SP165681). BRAZIL. Minas Gerais: Jaboticatubas, Serra do Cipó, km 2 of Estrada da Usina, 6-III-1972, A.B. Joly et al. CFSC 1260 (paratype SP165683). BRAZIL. Minas Gerais: Jaboticatubas, along road Lagoa Santa - Conceição do Mato Dentro Diamantina, Serra do Cipó, 6-III-1972, A.B. Joly et al. CFSC 1355 (paratype SP165682).

Panicum eitenii Renvoize, Kew Bull. 32(2): 424. 1978 (= Panicum molinoides Trin.). TYPE: BRAZIL. MinAs GeraIs: Mariana, at border with Ouro Preto, $6 \mathrm{~km}$ due SE of city of Ouro Preto, top of the

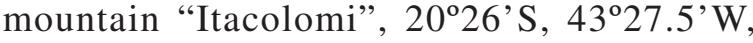
30-XI-1965, G. Eiten 7036 (holotype K, isotype SP140250).

Panicum kleinii Swallen, Sellowia. 18: 111. 1966. (= Panicum rude Nees). TYPE: BRAZIL. SANTA Catarina: Lauro Müller, Vargem Grande, R. Reitz \& R.M. Klein 7232 (paratype SP373932).

The above material is a fragment of a paratype housed at the United States National Herbarium (US2380657).

Panicum pirineosense Swallen, Phytologia 14(2): 78. 1966. (= Panicum chapadense Swallen). TYPE: BRAZIL. Goiás: Pirenópolis, at Pirineus, 18-X-1956, A. Macedo \& L.B. Smith 4805 (holotype US, isotype SP69328).

Panicum sendulskyi Zuloaga \& Morrone, Novon 6(3): 310, fig. 1. 1996. TYPE: BRAZIL. MINAS GERAIS: Jaboticatubas, along road Lagoa Santa Conceição do Mato Dentro - Diamantina, 9-XII-1971, T. Sendulsky et al. 414 (holotype SP318284).

Panicum soderstromii Zuloaga \& Send., Ann. Missouri Bot. Gard. 75(2): 446, fig.13. 1988. TYPE: 
BRAZIL. BAHIA: Jacobina, Serra do Brite, $11^{\circ} 09^{\prime}$ S, 4001'W, 21-VIII-1984, H.P. Bautista \& R.P. Orlandi 1000 (paratype SP204193).

Pariana ligulata Swallen, J. Wash. Acad. Sci. 30(2): 74, fig. 5. 1940. TYPE: BRAZIL. PARÁ: Boa Vista, Tapajós river, 7-13-I-1934, J.R. Swallen 3177 (holotype US, isotype SP337140).

Pariana sociata Swallen, J. Wash. Acad. Sci. 30(2): 76, fig. 6. 1940 (= Pariana gracilis Doell). TYPE: BRAZIL. Maranhão: between Caxias and Barra do Corda, 18-26-II-1934, J.R. Swallen 3599 (holotype US, isotype SP337146).

Paspalum ambustum Swallen, Phytologia 14(6): 366. 1967 (= Paspalum ovale Nees ex Steud.). TYPE: BRAZIL. Paraná: Ponta Grossa, Estação Experimental, 5-II-1946, J.R. Swallen 8466 (holotype US, isotype SP337152).

Paspalum biaristatum Filg. \& Davidse, Novon 4(1): 18, fig. 1. 1994. TYPES: BRAZIL. GoIÁs: Niquelândia, Macedo, 18 km N of Niquelândia, 10-VI-1992, T.S. Filgueiras 2341 (holotype IBGE, isotype SP373942). BRAZIL. GoIÁs: Niquelândia, Macedo, ca. 18 km N of Niquelândia, $14^{\circ} 18^{\prime}$ 'S, 48²3'W, 3-VIII-1992, T.S. Filgueiras \& R.D. Lopes 2408 (paratype SP373941). BRAZIL. GoIÁs: Niquelândia, Macedo, ca. $18 \mathrm{~km} \mathrm{~N}$ of Niquelândia, $14^{\circ} 18^{\prime}$ 'S, $48^{\circ} 23^{\prime}$ W, 3-VIII-1992, T.S. Filgueiras \& R.D. Lopes 2409 (paratype SP373940).

Paspalum chapadense Swallen, Phytologia 14(6): 363. 1967. TYPE: BRAZIL. MARANHÃo: between Caxias and Barra do Corda, 18-26-II-1934, J.R. Swallen 3508 (holotype US, isotype SP337136).

Paspalum compressifolium Swallen, Phytologia, 14(6): 381. 1967. TYPE: BRAZIL. Rio GRANDE Do Sul: Passo Fundo, Brigada Farm, 19-XII-1945, J.R. Swallen 7790 (holotype US, isotype SP337154).

Paspalum costellatum Swallen, Phytologia 14(6): 385. 1967. TYPE: BRAZIL. MARANHÃo: Carolina to Santo Antonio de Balsas, 20-25-III-1934, J.R. Swallen 3955 (holotype US, isotype SP36103).

Paspalum crustarium Swallen, Phytologia 14(6): 380. 1967. TYPE: BRAZIL. Maranhão: between Carolina and Santo Antonio de Balsas,
20-25-III-1934, J.R. Swallen 3985 (holotype US, isotype SP337137).

Paspalum dasytrichum Dusén ex Swallen var. glabrum L.B. Sm. \& Wassh., Bradea 2(35): 247. 1978. (= Paspalum quadrifarium Lam.). Type: BRAZIL. Santa Catarina: Lages, Coxilha Rica, 6-I-1946, J.R. Swallen 8173 (holotype US, isotype SP337144).

Paspalum eitenii Swallen, Phytologia 14(6): 385. 1967. (= Paspalum malacophyllum Trin.). TYPES: BRAZIL. MARANHão: Loreto, Ilha de Balsas, region between rivers Balsas and Parnaíba, about $40 \mathrm{~km}$ $\mathrm{S}$ of Loreto, place called "Picada", ca. $7^{\circ} 26^{\prime}$ 'S, 4507'W, 28-III-1962, G. Eiten \& L.T. Eiten 3901 (paratype SP85642). BRAZIL. MARANHÃO: Loreto, Ilha de Balsas, region between rivers Balsas and Parnaíba, about $30 \mathrm{~km}$ of Loreto, ca $1 / 2 \mathrm{~km} \mathrm{~N}$ of main house of São Raimundo Farm on trail to Santa Rita Farm, 7¹9'S, 4507-08'W, 6-IV-1962, G. Eiten \& L.T. Eiten 4078 (paratype SP85649). BRAZIL. MARANHÃO: Loreto, Ilha de Balsas, region between rivers Balsas and Parnaíba, about $30 \mathrm{~km}$ of Loreto, ca $1 / 2 \mathrm{~km} \mathrm{~N}$ of main house of São Raimundo Farm on trail to Santa Rita Farm, $7^{\circ} 19^{\prime}$ S, $45^{\circ} 07-08^{\prime}$ W, 6-IV-1962, G. Eiten \& L.T. Eiten 4089 (paratype SP85647). BRAZIL. PIauí: Fazenda Nacional, 3-IV-1934, J.R. Swallen 4207 (paratype SP36104).

Paspalum longiaristatum Davidse \& Filg., Novon 3(2): 129, fig. 1. 1993. TYPE: BRAZIL. GoIÁs: Niquelândia, Macedo, $18 \mathrm{~km} \mathrm{~N}$ of Niquelândia, 13-IV-1992, T.S. Filgueiras 2277 (holotype IBGE, isotype SP373939).

Paspalum niquelandiae Filg., Novon 5(1): 30, fig. 1. 1995. (= Paspalum longiaristatum Davidse \& Filg.). TYPE: BRAZIL. GoIÁs: Niquelândia, ca. $14^{\circ} 18^{\prime} \mathrm{S}, 48^{\circ} 23^{\prime} \mathrm{W}, 20 \mathrm{~km} \mathrm{~N}$ of Niquelândia, 19-V-1993, T.S. Filgueiras \& F.C.A. Oliveira 2461 (holotype IBGE, isotype SP367468).

The label of the herbarium sheet at SP shows, as collector, T.S. Filgueiras 2461 instead of T.S. Filgueiras \& F.C.A. Oliveira 2461, as indicated by Filgueiras (1995) in his original description.

Paspalum petrense A.G. Burm., Kew Bull. 35(2): 297. 1980. TYPE: BRAZIL. GoIÁs: ca. $20 \mathrm{~km} \mathrm{E}$ of Pirenópolis, Serra dos Pirineus, 8-IV-1979, T.S. Filgueiras \& A.G. Burman 430 (holotype K, isotype SP180113). 
Paspalum redondense Swallen, Phytologia 14(6): 388. 1967. TYPE: BRAZIL. PARANÁ: Guarapuava, Capão Redondo farm, 20-23-III-1946, J.R. Swallen 8837 (holotype US, isotype SP337134).

Paspalum splendens Hack., Oesterr. Bot. Z. 51(7): 238. 1901. TYPE: BRAZIL. Distrito Federal (Goí́s): Rio Gama, 24-IV-1895, A.F.M. Glaziou 22555 (sintype SP36115).

Paspalum swallenii Chase ex Swallen, Phytologia 14(6): 389. 1967. TYPE: BRAZIL. MARANHÃO: between Carolina and Santo Antonio de Balsas, 20-25-III-1934, J.R. Swallen 4100 (holotype US, isotype SP337138).

Paspalum usteri Hack., Repert. Spec. Nov. Regni Veg. 5(79-80): 1. 1908. TYPE: BRAZIL. SÃO Paulo: Perus, 25-III-1907, A. Usteri (holotype W, isotype SP9763).

Paspalum viale Swallen, Phytologia 14(6): 383.1967 (= Paspalum glaucescens Hack.). TYPE: BRAZIL. PARANÁ: near Curitiba, Campo Largo, 15-II-1946, J.R. Swallen 8591 (holotype US, isotype SP337135).

Pharus parvifolium G.V. Nash subsp. elongatus Judziewicz, Ann. Missouri Bot. Gard. 72(4): 874. 1985. TYPE: COSTA RICA. CARTAGO: $3 \mathrm{~km}$ E of Turrialba, "Los Espaveles" nature trail into a forested canyon of the River Reventazón, Centro Agronómico Tropical de Investigación y Enseñanza (C.A.T.I.E.), 954'N, 8339' W, 9-V-1983, $R$. Liesner et al. 15281 (holotype CR, isotype SP212493).

Piresia leptophylla Soderstr., Brittonia 34(2): 206, fig. 4. 1982. TYPES: BRAZIL. BAHIA: Una, Itapororoca Farm, ca. $10 \mathrm{~km} \mathrm{~S}$ of Una, 26-V-1976, T.R. Soderstrom et al. 2225 (holotype CEPEC, isotype SP235901). BRAZIL. BAHIA: Santa Cruz, km 5 road to Santa Cruz da Cabrália, ca. $16^{\circ} 15^{\prime} \mathrm{S}$, $39^{\circ} 05^{\prime} \mathrm{W}, 18-\mathrm{I}-1968$, C.E. Calderón 2047 (paratype SP224400). BRAZIL. BAHIA: Santa Cruz da Cabrália, Road Porto Seguro-Santa Cruz da Cabrália, $8 \mathrm{~km}$ NE from the road Eunápolis-Porto Seguro, ca. $16^{\circ} 20^{\prime} \mathrm{S}, 39^{\circ} 08^{\prime} \mathrm{W}, 2-\mathrm{IV}-1972$, C.E. Calderón \& R.S. Pinheiro 2197 (paratype SP223613). BRAZIL. BAHIA: Valença, Road from Valença to Guaibim, $12 \mathrm{~km} \mathrm{SW}$ of Guaibim beach, ca. $13^{\circ} 18^{\prime}$ 'S, 3901'W, 26-IV-1972, C.E. Calderón
\& R.S. Pinheiro 2244 (paratype SP223612).

Raddia angustifolia Soderstr. \& Zuloaga, Brittonia 37(1): 32, fig. 6. 1985. TYPE: BRAZIL. BAHIA: Itaju do Colonia, Uruguayana Farm and vicinity, 8 km S of Santa Cruz da Vitória, 4-IV-1976, T.R. Soderstrom et al. 2110 (holotype CEPEC, isotype SP373367).

Reitzia smithii Swallen, Sellowia 7: 8. 1956. TYPE: BRAZIL. SANTA CATARINA: Brusque, Azambuja, ca. 2706'S, 4854'W, III-1952, L.B. Smith \& R. Reitz 6123 (holotype US, isotype SP337141).

Schizachyrium crinizonatum S.T. Blake, Contrib. Queensland Herb. 17: 22, fig. 1F. 1974. TYPE: AUSTRALIA. NORTHERN TERritory: near (NW of) Katherine, 26-IV-1947, S.T. Blake 17477 (holotype BRI, isotype SP186178).

Streptostachys ramosa Zuloaga \& Soderstr., Smithsonian Contr. Bot. 59: 52, figs. 21-22. 1985. TYPE: BRAZIL. BAHIA: $22 \mathrm{~km} \mathrm{~S}$ of the Rio Galheirão, along highway BR-020, 8-IV-1976, G. Davidse et al. 12216 (holotype MO, isotype SP160930).

Steyermarkochloa unifolia Davidse \& R.P. Ellis, Ann. Missouri Bot. Gard. 71(4): 995, fig. 1. 1985 (1984). TYPE: COLOMBIA. GuAinía: near Coitara, ca. $7 \mathrm{~km} \mathrm{~S}$ of San Fernando de Atabapo (Venezuela), Western bank of the Rio Atabapo, $3^{\circ} 55^{\prime} \mathrm{N}, 67^{\circ} 43^{\prime} \mathrm{W}, 28-\mathrm{IV}-1979$, G. Davidse 16848 (paratype SP209986).

Stipa longicoronata Roseng. \& B.R. Arill., Bol. Fac. Agron. Univ. Montevideo 72: 20, tab. 4-5. 1964. TYPE: URUGUAY. Salto: Escuela de Agronomia, 1957, D. García 2150 (holotype MVFA, isotype SP98530).

Sucrea maculata Soderstr., Brittonia 33(2): 205, figs. 4-5. 1981. TYPE: BRAZIL. Espírito SANTO: Cachoeiro de Itapemirim, road from Cachoeiro de Itapemirim to Itabira, $20^{\circ} 21^{\prime} \mathrm{S}, 41^{\circ} 05^{\prime} \mathrm{W}$, 25-IV-1972, T.R. Sodestrom \& D. Sucre 1962 (paratype SP373366).

Sucrea monophylla Soderstr., Brittonia 33(2): 200, figs. 1-3. 1981. TYPES: BRAZIL. BAHIA: Itabuna, Ferradas, "Aberta Grande" Farm, ca. 14²7'21"'S, 39¹6’36”'W, 16-I-1968, C.E. Calderón 2045 (holotype CEPEC, isotype SP224328). BRAZIL. 
BAHIA: Ibicaraí, between Ibicaraí and Floresta Azul, at $7 \mathrm{~km}$ from Ibicarai, 3-IV-1976, T.R. Soderstrom et al. 2105 (paratype SP235891). BRAZIL. BAHIA: Itajuípe, ca. $50 \mathrm{~km} \mathrm{~N}$ of Itabuna, on BR 101, 20-IV-1976, T.R. Soderstrom et al. 2151 (paratype SP235866). BRAZIL. B AHIA: Ubaitaba, km 5 road Lages - Ubaitaba, ca. 14¹8'33'S, 39¹9'30'N, 13-I-1968, C.E. Calderón 2038 (paratype SP224416).

Thrasya crucencis Killeen, Ann. Missouri Bot. Gard. 77(1): 190, fig. 7. 1990. TYPE: BOLIVIA. SANTA Cruz: Nuflo de Chaves, Rancho Puesto Nuevo, $40 \mathrm{~km} \mathrm{~S}$ of Concepción, $16^{\circ} 25^{\prime} \mathrm{S}, 62^{\circ} 00^{\prime} \mathrm{W}$, 26-II-1987, T. Killeen 2334 (holotype ISC, isotype SP236499).

Thrasya glaziovii A.G. Burm., Brittonia 34(4): 460, fig. 2. 1982. TYPE: BRAZIL. Gorás: Santo Antonio do Descoberto, ca. $5 \mathrm{~km}$ from the city, 27-III-1980, T.S. Filgueiras 694 (holotype IBGE, isotype SP170039).

Thrasya longiligulata M. Bastos \& A.G. Burm., Bol. Mus. Paraense Emilio Goeldi, sér. Bot. 4(2): 236, figs. 1-2. 1988. TYPE: BRAZIL. PARÁ: Marabá, Serra dos Carajás, Mina Norte 1, 19-IV-1985, N.A. Rosa \& M.F.F. Silva 4691 (holotype MG, isotype SP373936).

Thrasya mosquitiensis Davidse \& A.G. Burm., Ann. Missouri Bot. Gard. 74(2): 434, fig. 1. 1987. TYPE: NICARAGUA. Zelaya: along banks and surrounding gallery forest of Rio Likas, near Silima Lila, ca. 14³0'N, 8350'W, 5-III-1979, J.J. Pipoly 4107 (holotype MO, isotype SP373365)

Thrasya oreophila A.G. Burm., Brittonia 34(4): 458, fig. 1. 1982. TYPE: BRAZIL. Minas GERAIS: São Gonçalo do Rio das Pedras, roadside descending into valley of Rio Jequitinhonha, 15-II-1981, A.G. Burman 591 (holotype SP185331, isotype SP358460).

Thrasya petrosa (Trin.) Chase var. ciliata A.G. Burm., Brittonia 32(2): 220, fig. 4. 1980. TYPE: BRAZIL. Minas Gerais: between the roads Diamantina - Extração and Diamantina - Minas Novas, 10-15 km from Diamantina, 10-12-IV-1979, A.G. Burman 454 (holotype SP180114).

Thrasyopsis rawitscheri Parodi, Bol. Soc. Argent. Bot. 1(4): 295. 1946. (= Thrasyopsis juergensii
(Hack.) Soderstrom \& A.G. Burm.). TYPE: BRAZIL. Paraná: Road Ponta Grossa - Tibagi, 14-II-1945, A.B. Joly 17 (holotype BAA?, isotype SP52310).

Trachypogon densus Swallen, Phytologia 14(2): 94. 1966. (= Trachypogon montufarii (Kunth) Nees). TYPE: BRAZIL. SANTA CATARINA: Lages, Fazenda de Criação, 4-I-1946, J.R. Swallen 8139 (holotype US, isotype SP337145).

Triraphis devia Filg. \& Zuloaga, Novon 9(1):36, fig. 1. 1999. TYPES: BRAZIL. GoIÁs: Chapada dos Veadeiros, Alto Paraíso, ca. $17 \mathrm{~km}$ from city to Colinas de Goiás, 1409'30' S, 47³9'54'W, 23-XI-1994, T.S. Filgueiras \& D. Alvarenga 3168 (holotype IBGE, isotype SP335437). BRAZIL. GoIÁs: Chapada dos Veadeiros, 17 km from Alto Paraíso / Colinas, 23-XI-1994, M.A. da Silva \& F.C.A. Oliveira 2382 (paratype SP280196).

Types not found at SP or with uncertain status:

Andropogon crispifolius Guala \& Filg., Nord. J. Bot. 15(1): 59, fig. 1. 1995. TYPE: BRAZIL. GoIÁs: Mineiros, Parque Nacional das Emas, ca. 10 km NE of the park headquarters, vicinity of Rio Jacuba, 27-V-1992, T.S. Filgueiras 2304 (holotype IBGE, isotype SP?).

Despite Guala \& Filgueiras (1995) pointed out an isotype at SP, we are unable to found it. There is a sheet of this species, at the herbarium SP, with the indication of isotype on its label, but the label also points 2305 as collector number, instead of 2304.

Arthoropogon filifolius Filg., Bradea 3(21): 151, fig. 1. 1981. TYPE: BRAZIL. Distrito Federal: Brasília, Estação Florestal "Cabeça do Veado", 15-IX-1980, T.S. Filgueiras 671 (holotype IBGE, isotype SP?).

Despite Filgueiras (1981) cited an isotype of this species at SP, the sheet at SP annotated as isotype is T.S. Filgueiras 761, instead of 671. At this moment it is not possible to know whether it is only a misprint.

Merostachys latifolia R.W. Pohl, Novon 2(2): 88, fig. 3. 1992. TYPE: NICARAGUA. JinotegA, s.d., W.D. Stevens \& J. Henrich 20418 (holotype ISC, isotype SP?).

Davidse \& Pohl (1992) indicated that there is an 
isotype of Merostachys latifolia R.W. Pohl at SP, but we are unable to find it.

Merostachys skvortzovii Send., Novon 5(1): 94, fig. 10. 1995. TYPE: BRAZIL. PARANá: Palmas, road Palmas-Ponte Serrado, 5-XII-1971, G. Hatschbach et al. 28267 (paratype SP?).

There are at SP many sheets which are types of Merostachys skvortzovii Send., but no duplicates of the material G. Hatschbach et al. 28267 were found, despite this was informed by Sendulsky (1995).

Olyra hoehnei Pilger, Repert. Spec. Nov. Regni Veg. 18: 122. 1922. (= Radiella esenbeckii (Steud.) C.E. Calderón \& Soderstr.). TYPE: BRAZIL. Minas Gerais: Serra do Caraça, I-1921, F.C. Hoehne s.n. (holotype SP5031).

Although Hoehne (1951) indicated even the registration number of this type, the herbarium material is probably lost.

Panicum cipoense Renvoize \& Send., Kew Bull. 35(1): 229, fig. 1. 1980. TYPE: BRAZIL. MinAs Gerais: Serra do Cipó, 3-XII-1949, A.P. Duarte 1999 (paratype SP?).

There is a holotype and some paratypes of this species at SP, but the sheet A.P. Duarte 1999 was not found, despite the information given by Renvoize \& Sendulsky (1980).

Streptostachys rigidifolia Filg., Morrone \& Zuloaga, Novon 3(3): 252, fig. 1-3. 1993. TYPE: BRAZIL. MARANHÃo: Loreto, BR 230 road to São Raimundo das Mangabeiras, ca. 22 km from São Raimundo, 6056'S, 45ํ'19'W, 13-IV-1988, L.B. Bianchetti et al. 634 (holotype CEN, isotype SP?).

An isotype of this species is pointed for SP by Filgueiras et al. (1993b) but we were unable to find it.

\section{Aknowledgements}

To Regina T. Ninomya for her assistance during the study of the type collection of Poaceae. The second author acknowledge CNPq (National Council for Scientific and Technological Research) for the grant received (process 303962/2004-6).

\section{Literature cited}

Davidse, G. \& Pohl, R.W. 1992. New taxa and nomenclatural combinations of Mesoamerican grasses (Poaceae). Novon 2: 81-110.

Filgueiras, T.S. 1981. Species duae Graminearum Brasiliae novae. Bradea 3: 151-155.

Filgueiras, T.S. 1995. Paspalum niquelandiae (Poaceae: Paniceae), a new species from the serpentine outcrops of Central Brazil. Novon 5: 30-33.

Filgueiras, T.S., Davidse, G. \& Zuloaga, F.O. 1993a. Ophiochloa, a new endemic serpentine grass genus (Poaceae: Paniceae) from the Brazilian Cerrado vegetation. Novon 3: 360-366.

Filgueiras, T.S., Morrone, O. \& Zuloaga, F.O. 1993b. A new species of Streptostachys (Poaceae: Paniceae) from Brazil. Novon 3: 252-257.

Filgueiras, T.S., Davidse, G., Zuloaga, F.O. \& Morrone, $O$. 2001. The establishment of the new genus Altoparadisium and reevaluation of Arthropogon (Poaceae: Paniceae). Annals of the Missouri Botanical Garden 88: 351-372.

Guala, G.F. \& Filgueiras, T.S. 1995. Andropogon crispifolius (Poaceae: Andropogonae): a new species from the cerrado of central Brazil. Nordic Journal of Botany 15: 59-62.

Hoehne, F.C. 1937. Resenha histórica para a commemoração do vigésimo anniversário da Secção de Botânica e Agronomia annexa ao Instituto Biológico de São Paulo. Directoria de Publicidade Agrícola, Secretaria da Agricultura Indústria e Commércio do Estado de São Paulo, São Paulo.

Hoehne, F.C. 1951. Dados autobio-bibliográficos do botânico F. C. Hoehne até 31-12-1950. Relatório Anual do Instituto de Botânica, referente ao exercício de 1950. Instituto de Botânica, São Paulo, pp. 63-153.

Mamede, M.C.H. 1996. Catálogo dos tipos de fanerógamas do Herbário do Estado "Maria Eneyda P. K. Fidalgo", Instituto de Botânica, São Paulo, Brasil. Boletim do Instituto de Botânica 10: 233-325.

Renvoize, S.A. \& Sendulsky, T. 1980. A new species in Panicum group Lorea (Gramineae) from Brazil. Kew Bulletin 35: 229-230.

Sendulsky, T. 1995. Merostachys multiramea (Poaceae: Bambusoideae: Bambuseae) and similar species from Brazil. Novon 5: 76-96.

Zanin, A. \& Longhi-Wagner, H.M. 2003. Taxonomic novelties in Andropogon (Poaceae - Andropogoneae) from Brazil. Novon 13: 368-375. 